\title{
Estimating the impact of unsafe water, sanitation and hygiene on the global burden of disease: evolving and alternative methods
}

\author{
Thomas Clasen ${ }^{1,2}$, Annette Pruss-Ustun ${ }^{3}$, Colin D. Mathers ${ }^{3}$, Oliver Cumming ${ }^{2}$, Sandy Cairncross ${ }^{2}$ and John M. \\ Colford Jr ${ }^{4}$
}

1 Department of Environmental Health, Rollins School of Public Health, Emory University, Atlanta, GA, USA

2 Department of Disease Control, Faculty of Infectious and Tropical Diseases, London School of Hygiene and Tropical Medicine,

London, UK

3 World Health Organization, Geneva, Switzerland

4 Department of Epidemiology, School of Public Health, University of California, Berkeley, CA, USA

\begin{abstract}
The 2010 global burden of disease (GBD) study represents the latest effort to estimate the global burden of disease and injuries and the associated risk factors. Like previous GBD studies, this latest iteration reflects a continuing evolution in methods, scope and evidence base. Since the first GBD Study in 1990, the burden of diarrhoeal disease and the burden attributable to inadequate water and sanitation have fallen dramatically. While this is consistent with trends in communicable disease and child mortality, the change in attributable risk is also due to new interpretations of the epidemiological evidence from studies of interventions to improve water quality. To provide context for a series of companion papers proposing alternative assumptions and methods concerning the disease burden and risks from inadequate water, sanitation and hygiene, we summarise evolving methods over previous GBD studies. We also describe an alternative approach using population intervention modelling. We conclude by emphasising the important role of GBD studies and the need to ensure that policy on interventions such as water and sanitation be grounded on methods that are transparent, peer-reviewed and widely accepted.
\end{abstract}

keywords global burden of disease, water, sanitation

\section{Introduction}

The 2010 global burden of disease (GBD) study led by the Institute for Health Metrics Evaluation (IHME) reflects major changes from previous estimates for some categories of diseases and injuries and their corresponding risk factors. One area experiencing large declines was in diarrhoeal diseases and the impact of unsafe water, sanitation and hygiene (WASH) on these diseases. Compared with 1990, mortality from diarrhoeal diseases is reported to have fallen by $41.9 \%$ ( $49 \%$ on an ageadjusted basis) from 2.5 million to 1.4 million in 2010 (Lozano et al. 2012). Unimproved water and sanitation, which accounted for an estimated $6.8 \%$ of disabilityadjusted life years (DALYs) in 1990 and 3.7\% in 2000, was estimated to represent only $0.9 \%$ of the DALYs in 2010 (Lim et al. 2012). Among risk factors, the rank of unimproved sanitation fell from 15 th to 26 th and that of unimproved water from 22 nd to 34 th.
The dramatic reductions in the estimates for diarrhoea are consistent with declines in certain other childhood diseases and with recent trends and projections (Lim et al. 2012; Lozano et al. 2012). Among childhood diseases, the 2010 estimates also reflect major declines in DALYs associated with lower respiratory infections (from 3.4 to 2.8 million), neonatal disorders (from 3.1 to 2.2 million), measles (from 0.63 to 0.13 million) and tetanus (from 0.27 to 0.06 million) (Lozano et al. 2012). In addition to improved WASH, reductions in these diseases were attributable in part to lower risks from childhood underweight, suboptimal breastfeeding and micronutrients deficiencies. They and the declining risk associated with inadequate WASH are also consistent with the broader shift from age-specific communicable, maternal, neonatal and nutritional causes towards non-communicable diseases.

Despite these apparent health gains, however, the latest estimates were received with some scepticism among 
long-time implementers and advocates of WASH interventions (Watts \& Cairncross 2012). One reason is the geographical heterogeneity in the estimates: despite the substantial drop in global rank, unimproved sanitation and unimproved water remain among the top 12 risk factors in most of sub-Saharan Africa where diarrhoea remains a leading killer (Liu 2012). More than this, however, there is continuing uncertainty about the methods used in estimating the risks associated with deficiencies in WASH. This uncertainty is reflected in the evolution of the methods employed over the successive estimates over the last three decades (Lopez 2005). It has been aggravated recently by inadequate consultation and publication of the overall results without a detailed description of the underlying methods (Watts \& Cairncross 2012).

This paper summarises the evolving methods to assess the burden of disease and risks associated with inadequate WASH. To demonstrate the range of methodologies available for estimating disease burden, we also summarise an alternative approach using population intervention modelling. The aim of this paper was to provide a context for companion papers proposing alternative assumptions and methods which may yield different estimates of the burden of disease and risk factors associated with deficiencies in WASH or confirm the current estimates.

\section{Global Burden of Disease Study}

Most countries have been compiling data on mortality and the cause of death for decades; London and some other cities have been doing so for centuries (Farr 1885). Over the last century, the systems for monitoring morbidity and mortality have evolved, with registries of specific diseases, improved standardization and increasing details on the exposures and other risk factors (Lopez 2005). Compiling these data at an international level offers important benefits, allowing health authorities to compare their results with other countries, explore opportunities for reducing disease, and establish goals and identifying effective intervention strategies that can be implemented locally.

The World Health Organization (WHO) undertook the first GBD Study in the early 1990s in collaboration with the World Bank and the Harvard School of Public Health (Murray \& Lopez 1996). Its aim was to assess the mortality and morbidity associated with 131 leading diseases and injuries; it also sought to characterise the contribution of 10 specified risk factors to this disease burden. The results were used by the World Bank to estimate the cost-effectiveness of various interventions for more than 100 countries at different stages of development as of
1990 (World Bank 1993). It represented the first internally consistent set of epidemiological estimates covering all age groups on a global scale. It drew attention to the scope of widespread communicable causes such as malaria, tuberculosis and diarrhoea, but also highlighted the large and growing disease burden associated with mental health, non-communicable diseases and injuries.

The 1990 GBD Study incorporated some major innovations in assessing disease burden. Perhaps best known of these was the use of DALYs as a common metric that includes both premature mortality (years of life lost, or YLLs) and years of life lived with a disability weighted for its severity (YLDs) (Murray 1996). The 1990 GBD relied for its estimates of morbidity and mortality on a large range of data available at the country level, often with varying levels of reliability and completeness, and often requiring bold extrapolation. To estimate the attributable burden, the study compared 'the difference between the currently observed burden and the burden that would be observed if past levels of exposure had been equal to a specified reference distribution of exposure' (Murray \& Lopez 1997).

To estimate the impact of eliminating risk factors, researchers relied on epidemiological evidence and expert opinions. For 'poor water supply, sanitation and personal and domestic hygiene', one of the 10 risk factors considered, the 1990 GBD Study commissioned a review by Huttly et al. (1997). Estimates were derived from the product of the estimated efficacy of the interventions and the proportion of the burden of disease that occurs among the exposed. The estimated 'reduction achievable through feasible interventions' was $40 \%$ for diarrhoea (which included diarrhoea, dysentery, cholera and typhoid), 30\% for trachoma, 30\% for the 'tropical cluster' (schistosomiasis, South American trypanosomiasis, and Bancroftian filariasis), and $40 \%$ for intestinal worms. Because of the unavailability of exposure estimates, the hygiene estimate was taken to support outcome-based estimates and to define uncertainty intervals only (Lim et al. 2012). The study found that the WASH risk factor ranked second (after malnutrition), causing an estimated 128 million DALYs (99 million from diarrhoea, 18 million from intestinal worms, 8 million from the tropical cluster, and 3 million from trachoma) or $6.8 \%$ of all DALYs (World Bank 1993).

\section{The 2000-2004 GBD Study}

The 1990 GBD Study represented a major advance in the quantification of diseases, injuries and risk factors on a global and regional basis. Nevertheless, even its lead authors acknowledged the need for improvements, 
including the method for risk factor assessment (Murray \& Lopez 1999). Among other things, there was an acknowledgement of the need for stronger epidemiological evidence for the causal associations between risk factors and health outcomes.

In 1998, the Disease Burden Unit was organised at the WHO and charged with the preparation of estimates of the global burden of disease and injuries for the year 2000. Subsequently, GBD estimates were updated annually for years 2000-2002 and were published in the WHO's annual World Health Reports, followed by a stand-alone report for the year 2004 (WHO 2008). The new GBD Study (hereinafter, the '2000-2004 GBD Study') incrementally revised and updated estimates of incidence, prevalence and YLD for non-fatal health outcomes. By the time of the GBD 2004 study (WHO 2008), 97 of the 136 causes had been updated, including all causes of public health importance or with substantive YLD contribution to DALYs.

In addition to seeking more reliable data, the 20002004 GBD Study reflected major changes in assessing risk factors (Ezzati et al. 2004). The new framework sought to measure changes in the attributable proportions of cause-specific disease burden expected under different levels of population exposure (Murray \& Lopez 1999). Attributable fractions of disease associated with each risk factor were established by comparing the current estimated distribution of exposure with a counterfactual distribution defined as the distribution leading to the lowest theoretical level of disease burden. These theoretical minima were developed by expert groups for 26 risk factors as part of the WHO Comparative Risk Assessment (CRA) study (Ezzati et al. 2002).

For unsafe water and sanitation, the reference outcome was diarrhoea (although the outcome was defined to include typhoid, paratyphoid, schistosomiasis, trachoma, ascariasis, trichuriasis, hookworm infection and dracunculiasis) and the theoretical minimum was no transmission of diarrhoeal diseases through water, sanitation or hygiene (Pruss et al. 2002). Other potentially waterborne diseases (e.g. hepatitis A and E, arsenicosis and flurosis) and diseases transmitted by vectors that breed in water (e.g. dengue, malaria) were excluded due to insufficiency of information to estimate attributable fractions. Estimates for sources of exposure were based on the Global Water and Sanitation Assessment (2000) assembled by the WHO/UNICEF Joint Monitoring Committee on Water and Sanitation using household-levels surveys.

In assessing risk associated with WASH, the 20002004 GBD Study adopted a new framework that included six different exposure scenarios ranging from regulated piped water and sanitation with hygiene to a lack of improved water supply or improved sanitation (Figure 1) (Ezzati et al. 2002; Pruss et al. 2002). These were then combined with hazard estimates obtained from systematic reviews and individual studies. A relative risk of 1.0 was assigned to exposure scenario I, representing the minimum theoretical exposure (i.e no disease transmission through water and sanitation). Transitioning from scenario I to scenario II (regulated water supply) carried a relative risk of 2.5. Scenario III was characterised by three subcategories: improved drinking water

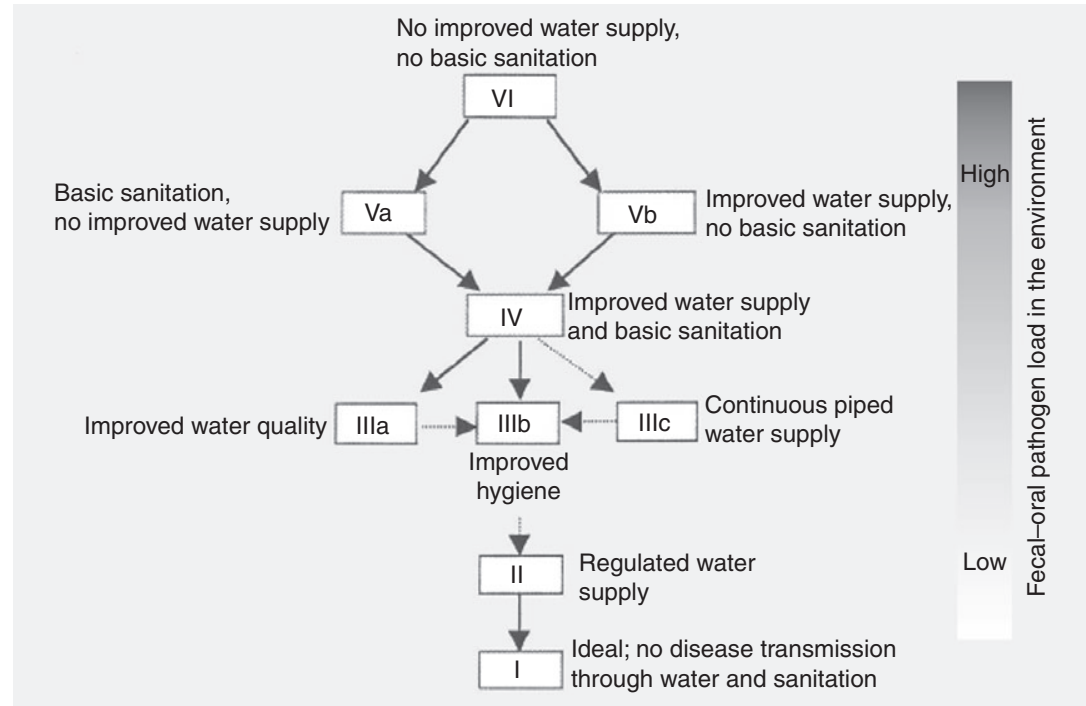

Figure I Water, sanitation and hygiene risk scenarios (from Pruss et al. 2002; ). 
quality (IIIa) represented by drinking water disinfected at point of use (POU); improved personal hygiene (IIIb); and improved access to drinking water (IIIc) generally represented by water piped to the household. Once again, owing to the lack of exposure estimates, hygiene was considered only to define the likely intervals of risk reductions (Lim et al. 2012). Significantly, the 2000 GBD Study assumed on the basis of existing epidemiological evidence that there was a reduction in risk associated with each of these improvements when compared simply to improved water supply and improved sanitation (IV) (Pruss et al. 2002). Thus, the absence of these conditions added to the overall burden associated with inadequate WASH beyond the higher risk scenarios, improved sanitation without improved water (Va), improved water without improved sanitation ( $\mathrm{Vb}$ ) and neither (VI).

Notably, none of the studies included in developing the risk estimates were blinded. This is true even though blinded trials of POU water quality interventions had already been undertaken, all showing no protective effect on diarrhoea or gastroenteritis from improvements in drinking water quality (Kirchhoff et al. 1985; Austin 1993; Hellard et al. 2001; Colford et al. 2002) . Instead, the 2000-2004 GBD Study relied on two unblinded trials (Semenza et al. 1998; Quick et al. 1999) , using 44.7\% as its estimate of the risk reduction associated with improved drinking water quality (scenario IV-II). Subsequent reviews also raised questions about the interpretation of the early blinded trials, either due to methodological quality or the fact that they were conducted in settings with good water quality (Clasen et al. 2006).

In 2008, the WHO Department of Health Statistics and Informatics published GBD Study for the year 2004, updating estimates and providing more regional breakdowns (WHO 2008). For diarrhoeal disease deaths, it used death registration data where available; for those without such data, the estimates were based on a regression model to estimate proportional mortality (BoschiPinto et al. 2008). Associated risk factors were updated in a separate publication (WHO 2009). The 2004 GBD Study estimated a total of 2.2 million deaths annually from diarrhoeal diseases, representing the fifth leading cause of mortality and $3.7 \%$ of all deaths. This disease burden fell mainly on low-income countries (1.8 million deaths) particularly in Africa and Southeast Asia; diarrhoeal diseases accounted for $17 \%$ of deaths among children under five (WHO 2008). The 2004 GBD Study also estimated more than 4.4 billion episodes of diarrhoeal disease, yielding a combined disease burden of 72.8 million DALYs $(4.8 \%$ of the total), second only to lower respiratory infection (94.5 million, $6.2 \%$ ).
For estimating the role of risk factors, the 2004 GBD Study employed the same framework developed for the 2002 World Health Report, but focused solely on diarrhoea as an outcome (WHO 2009). For water and sanitation, exposure estimates were from the WHO/UNICEF Joint Monitoring Programme for Water Supply and Sanitation using coverage data from 2004. Relative risks for diarrhoea were from the CRA 2000 study. Based on this approach, the 2004 Study reported that unsafe water, sanitation and hygiene caused 1.9 million deaths annually, or 3.2\% of global mortality (WHO 2009). Combined with morbidity figures, unsafe water, sanitation and hygiene were the fourth leading contributor to the GBD, representing 64 million DALYs or $4.2 \%$ of the total.

\section{The 2010 GBD Study}

In 2007, leading GBD researchers moved to the newly organised IHME, a research centre supported by the Bill \& Melinda Gates Foundation and housed at the University of Washington. Together with its academic collaborators and scores of other researchers, the IHME published the 2010 GBD estimates in a series of papers and abstracts in The Lancet starting in December 2012 (the 2010 GBD Study). WHO was a collaborator in work leading to the GBD 2010 results, but did not endorse all the results. In some areas, the results of the GBD differ substantially from analyses carried out by WHO and other United Nations agencies. In many other areas, the GBD results update and are broadly similar to previous WHO analyses. WHO is continuing to collaborate in some areas of disease burden work, and it is anticipated that the GBD 2010 results will contribute to revisions for WHO global health estimates in 2013.

While the new study made adjustments to disability factors, it did not change the scope of diseases associated with WASH despite other evidence of risk associated with deficiencies in WASH. Thus, it still does not include malnutrition despite estimates of 860000 deaths annually from malnutrition from unsafe water, inadequate sanitation and insufficient hygiene (Pruss-Ustun et al. 2002). A recent Cochrane Review found some evidence of a small effect on stunting of certain WASH interventions although all studies were of poor quality (Dangour et al. 2013). Environmental (tropical) enteropathy, a widespread condition associated with poor sanitation and hygiene and believed to contribute significantly to child stunting and underweight (Humphrey 2009), is also not part of the current estimates despite recent evidence of its adverse impact on health in WASH-poor settings (Lin et al. 2013). 
There was, however, a major change in assessing the impact of WASH-related risk factors. Like the 2000 GBD Study, the 2010 GBD Study follows the approach of assigning risk ratios to the different exposure scenarios. In general, these are drawn from published meta-analyses or updated meta-analyses undertaken as part of this study. Significantly, however, the 2010 Study undertook special analyses to support risk estimates with respect to water and sanitation (Murray et al. 2012). According to the 2010 GBD Study, this was motivated in the case of water and sanitation by conflicting evidence on the effect of water quality interventions to prevent diarrhoeal diseases (Lim et al. 2012). The new review does not included hygiene.

As of December 2013, only the abstract on water and sanitation meta-analysis used for the 2010 GBD Study has been published (Engell \& Lim 2013). Accordingly, the methods are not publicly available. The review is described as an update of previous reviews by Cairncross et al. (2010), Waddington et al. (2009), Fewtrell et al. (2005) and Clasen et al. (2010). One important departure from these previous reviews was the inclusion of observational studies, increasing the number of comparisons although potentially compromising methodological quality. Like previous reviews (Clasen et al. 2006; Waddington et al. 2009), POU water interventions were subgrouped based on blinding. Indicator variables were included for whether the baseline condition represented improved or unimproved water sources or sanitation as potential covariate to account for heterogeneous control groups. Subgroup analysis was used to investigate differences in effect by age.

The review found significant protective effects both for improved water sources (RR 1.34, 95\% CI 1.02-1.72) and for improved sanitation (RR 1.33, 95\% CI 1.021.74). It found no protective effect from piped water or source water treatment compared with improved water supply $(P=0.50$ and $P=0.65$, respectively) or by age $(P=0.19)$. Like previous reviews, Engel \& Lim found no effect from POU water quality interventions when blinding was considered $(P=0.08)$. Based on this, the 2010 GBD Study concluded that there was no risk associated with water quality for water supplies that are 'improved' as prescribed by the WHO/UNICEF Joint Monitoring on Water and Sanitation (JMP): these consist of protected wells or springs, public standposts, tube wells, boreholes and rainwater collection.

The difference in pooled estimates of effect between blinded and open trial designs has been well documented (Clasen et al. 2006; Schmidt \& Cairncross 2009; Waddington et al. 2009). A more recent large-scale placebocontrolled trial also reported no protective effect from a chlorine tablet intervention (Boisson et al. 2013). Nevertheless, other researchers have not asserted that these results imply that water quality interventions are ineffective or that there is no additional benefit from ensuring drinking water quality. A detailed analysis of each of the blinded studies raises questions about the methodological quality of many of the studies, including whether they were in fact adequately blinded (Clasen et al. 2006). Studies were generally small or of short duration (Kirchhoff et al. 1985; Jain et al. 2009) and were conducted in settings where the ambient water quality either met WHO guidelines or where contamination was low (Colford et al. 2002; Hellard et al. 2001, Jain et al. 2010) or used placebos that were or may not have been neutral (Austin 1993; Boisson et al. 2010). Boisson et al. (2013) reported poor and inconsistent uptake of the intervention and a modest impact on water quality, so that the lack of a protective effect is consistent with epidemiological modelling of water quality interventions (Brown \& Clasen 2012; Enger et al. 2013). Studies in low-income countries are generally conducted in settings where poor sanitation and hygiene create other sources of exposure that may neutralise any benefit from improved water quality alone.

While calling for more blinded studies, other researchers have suggested an alternative approach that discounts the results of open trial designs of self-reported outcomes (Clasen et al. 2006). In a systematic review comparing the results of blinded trials with open trials of subjective outcomes (such as self-reported diarrhoea), Wood et al. (2008) found the latter to exaggerate effect estimates by $25 \%$ (95\% CI: 7-39\%). A more recent review concluded that lack of, or unclear, double blinding (compared with effective double blinding) was associated with an average $28 \%$ exaggeration of intervention effects in the case of trials of subjective outcomes (Savović et al. 2012). Using this approach to discount the results of open trial designs, Hunter (2009) found that some POU water quality interventions were nevertheless protective, suggesting a continuing underlying risk from untreated water.

The results of the meta-analysis by Engel and Lim had a significant impact on the risk estimates associated with WASH interventions for purposes of the 2010 GBD Study. Because it found no added benefit from household connections over other 'improved water supplies' or from water quality interventions alone (based on blinded POU interventions), the authors restricted their analysis to a comparison between improved $v s$. unimproved water and improved $v s$. unimproved sanitation - in each case based on the JMP definitions. This is in sharp contrast to previous GBD estimates which are based on additional reductions in risk from improved and continuous water quality 
and improved hygiene (scenario III) and regulated water supply (scenario II) before arriving at the optimal level represented by the counterfactual (scenario I). By abandoning the risk scenarios used in previous GDB estimates in favour of a simple 'improved/unimproved' dichotomy, the 2010 GDB Study loses key aspects of exposure such as water quantity, quality, access, continuity and reliability.

One final issue concerning the risk factors associated with WASH is the assumption that 'improved' water supplies are safe. The 'theoretical minimum-risk exposure distribution' is based on 'all households use improved water source' (Lim et al. 2012). There is substantial evidence from field studies that most types of water sources that meet the definition of 'improved' - including protected wells and springs, public taps or standpipes, boreholes and tube wells - are contaminated with faecal pathogens and do not meet the guideline values included in the WHO Guidelines for Drinking-water Quality (Onda et al. 2013). The JMP, whose data were used as the basis for the exposure estimates, acknowledges that the dichotomous improved/unimproved typology focussed on service level is a poor proxy for water quality (WHO/ UNICEF 2012).

\section{A way forward? Population intervention (and other) models for the estimation of burden of disease}

Earlier approaches - and their limitations - to the problem of estimating the burden of disease attributable to risk factors associated with WASH have been detailed above. One basic problem is that it can be difficult to obtain outcome data under a sufficiently broad range of exposure experience in a large population. This difficulty is exacerbated when impact estimation is made using intention to treat analysis results from randomised trial data. Such data are necessarily restricted to the range of exposures experienced by the enrolled (and likely atypical) population and the specific interventions used in that one trial. This restriction renders randomised trials unable to inform the estimation of population-attributable fractions without significant extrapolation beyond the context in which they were conducted.

This problem might be re-framed in an epidemiologic context as a question about how best to quantify disease outcomes causally related to the impact of potential improvements in WASH risk factors. Specifically, given the risk factor exposure distribution and disease outcome experience of a specific population, how can one measure the 'counterfactual' experience that would be expected in this same population if the exposure distribution were changed? This counterfactual distribution of exposures might, theoretically, be defined as a scenario in which all of the risk factors are eliminated. Alternately, the counterfactual scenario might be a scenario in which the population exposure to risk factors is only reduced to a defined (and realistically achievable) level for that specific population.

This problem of estimation under a counterfactual exposure scenario is not unique to the WASH sector. Theoretical and applied work in epidemiology in the last decade has resulted in approaches that evaluate the impacts of potential changes in exposures in a population (arising from interventions targeted to those exposures). In particular, these approaches can take advantage of observational data in settings where randomised control data are not available or are not optimal. One of these approaches, nested broadly within the area of causal inference research, is referred to as a 'population intervention model' (Hubbard \& van der Laan 2008, Ahern et al. 2009; Fleischer et al. 2010).

In a population intervention model, the prevalence of a disease outcome in an observed population is compared to the disease prevalence in a population under a counterfactual exposure distribution. This counterfactual population is constructed as if all factors except the exposure of interest are held to the same levels as found in the observed population. The exposure of interest is either changed to a different level (or removed completely), and the prevalence of disease in the counterfactual population with the altered exposure distribution is then re-estimated by the model. The change in disease prevalence (under the observed vs. the counterfactual exposure distribution) provides an estimate of the burden of disease attributable to the exposure. Population intervention models employ the same statistical estimation techniques as marginal structural models (Robins et al. 2000; Van der Laan \& Rose 2011), but they target a population-attributable fraction parameter rather than other alternatives such as a risk difference or a risk ratio.

In applied terms, the exposure distribution and the prevalence of a disease outcome in an observed population are estimated using the best available data for a specific country or population. Ideally, these data will include exposure and disease outcome information for a sufficiently large sample of subjects, drawn from a representative sample of the population, across the full range of the exposure distribution. As has been described above for the earlier GBD estimates, the observed data may be available from some combination of national censuses, other surveys used by the JMP and/or other country-specific surveys conducted in a rigorous manner with random sample selection. 
The population intervention model provides an estimate of the prevalence of disease under the specific counterfactual exposure distribution scenario(s) of interest. The population intervention model is not overly difficult to implement with appropriate biostatistical support. The technical details for these models have been described by Greenland and Drescher (1993) using maximum likelihood techniques and Hubbard and van der Laan (2008) using direct estimation. The results from these models could be expressed in terms of DALYs or with any other measure derived from estimates of disease using standard methods.

Population intervention models are not a panacea for the many difficulties that arise in the estimation of disease burden. These models do, however, provide a number of potential advantages. First, estimation of the effect of a change in exposure distribution is made directly on the population of interest in a specific country or region; unlike the current GBD approach, it is not necessary to invoke a relative risk estimate derived from meta-analysis of trials or observational studies in other countries or areas of the world, which often enrol non-representative segments of the target population. Such broadly synthesised data from widespread sources may have limited relevance in individual countries. Second, the population intervention model approach can be applied to estimate the burden of disease in any country from which properly sampled exposure and disease outcome data are available (generally, these will be properly constructed samples correctly weighted for the sampling design). Third, the burden of disease calculations could estimate separate population intervention models for morbidity and mortality as many large-scale, population-based surveys measure mortality - this would avoid the need to assume that counterfactual scenarios estimated from field studies that measure morbidity as their outcome translate directly to effects on mortality. Finally, population intervention models permit the incorporation of important covariates into the analysis and are limited only by the available data. If, for example, one is interested in using observational data to estimate a counterfactual scenario in which sanitation is improved, an investigator might wish to control for socio-economic status as a potential confounder. If information on socio-economic status is available, it (and other measured potential confounders) could be controlled for during estimation of diarrhoea risk under the counterfactual scenario.

The population intervention model approach is not without limitations. To establish a useful counterfactual population, this approach depends upon having sufficient data about exposure and disease levels for the relevant portions of the population that meet the definition of the counterfactual. For example, if the counterfactual of interest involves interventions that move a population from poor water quality to good water quality (however defined), it is necessary to have information about the rate of disease in both of the subgroups of the population receiving poor and good quality water in that country. Additionally, although large population health surveys measure many exposures and outcomes, they seldom include all outcomes of interest. An additional limitation is that if spillovers are unaccounted for, this approach might underestimate the total impact of interventions. For example, even if the population data contain sufficient information about individuals living under the counterfactual exposure condition of interest (e.g. good water quality), it is possible that estimates from the population intervention model would be biased towards the null if reduced disease outcomes among neighbouring individuals due to the intervention (i.e. spillovers in the same direction as the treatment effect) were not taken into account. The latter two limitations, unmeasured outcomes of interest and unmeasured spillover effects, however, may also be limitations of the current GBD approach. Finally, the population intervention models described above are constructed by changing the exposure of interest to a different (fixed) level. Recent developments in the field have introduced flexibility to this approach by allowing for a change in the exposure of interest to a counterfactual distribution of exposures rather than a fixed level of exposure. This 'stochastic intervention' approach is described by Muñoz and van der Laan (2012).

Population (and stochastic) intervention models might best be considered as complementary tools whose results could be compared to currently used approaches for GBD estimation in the specific countries for which adequate data are available. Where the current approaches arrive at estimates consistent with the population intervention model, an added degree of confidence may be possible. Situations in which the two approaches significantly differ in their estimation of the burden of disease suggest a need for further exploration of the limitations and strengths of the data used for each approach.

\section{Conclusion}

Estimates of the global burden of disease and the risk factors associated therewith have become an essential tool in public health, providing critical information for monitoring progress and setting priorities. Results over the past three decades have identified important trends, including an overall reduction in child mortality and a reduced role of communicable, maternal, neonatal and nutritional 
causes. Consistent with these trends and overall improvements in water, sanitation and hygiene, successive estimates have shown reductions in the disease burden from faecal-oral diseases such as diarrhoea and in the risk associated with inadequate WASH.

Like previous iterations of the GBD Study, the 2010 Study incorporates important changes in methods. This is part of a continuing evolution designed to improve estimates. This evolution will continue, as will efforts to explore alternative approaches such as the population intervention model. These alternatives also allow for triangulation to improve the reliability of estimates.

Such alternatives may be able to address some of the major shortcomings of current approaches to estimate the disease burden associated with WASH. These shortcomings include ongoing challenges that GBD estimates still fail to address, such as projecting results from morbidity studies to mortality, which represents most of the disease burden from faecal-oral diseases such as diarrhoea. There are also issues in deriving attributable fractions from research-driven efficacy studies in settings carefully selected for their adverse conditions, as well as from effectiveness studies where compliance may be poor. New methods are needed to define and capture important characteristics of exposures that go beyond the crude 'improved/unimproved' dichotomy. For example, the JMP is currently piloting methods to assess water quality in the field as part of national surveys. New methods or data are also necessary to deal with the lack of risk estimates from poor hygiene, which continues to be omitted from GBD studies due to the lack of exposure data.

In respect of the water and sanitation risk factors, however, the 2010 GBD Study reflects changes that resulted not only from new methods but also from new interpretations of existing epidemiological evidence - particularly in respect of the contribution of water quality. Unfortunately, as discussed above, the systematic review on which these new interpretations are based has not yet been published. Thus, while the new estimates have dramatically changed the relative role of inadequate water and sanitation as contributors to the global burden of disease, it is not clear whether the estimates are more reliable.

Because GBD studies have a substantial impact on the allocation of scarce resources, both at a national and international level, it is important that policymakers, implementers and funders have confidence in them. It is also important that changes in priorities cannot be driven in the short term by changes in the underlying approaches for developing estimates unless those changes are transparent, comprehensively reviewed in advance of implementation and generally agreed as an advance over previous approaches. This is particularly true for the disease burden impacted by long-term and widespread interventions such as the infrastructural and other investments and commitments necessary to achieve improvements in water, sanitation and hygiene.

\section{Acknowledgements and Disclaimer}

The authors gratefully acknowledge the contributions of Jennifer de France, Jamie Bartram, Ben Arnold and Jade Benjamin-Chung in commenting on this paper. This work was supported in part by the World Health Organization. The authors alone are responsible for the views expressed in this publication, and they do not necessarily represent the decisions or policies of their respective institutions, including the World Health Organization.

\section{References}

Ahern J, Hubbard A \& Galea S (2009) Estimating the effects of potential public health interventions on population disease burden: a step-by-step illustration of causal inference methods. American Journal of Epidemiology 169, 1140-1147.

Austin CJ (1993). Investigation of in-house water chlorination and its effectiveness for rural areas of the Gambia. Dissertation, Tulane University School of Public Health and Tropical Medicine, New Orleans, LA.

Boisson S, Kiyombo M, Sthreshley L, Tumba S, Makambo J \& Clasen T (2010) Field assessment of a novel household-based water filtration device: a randomised, placebo-controlled trial in the Democratic Republic of Congo. PLoS One 5, e12613.

Boisson S, Stevenson M, Shapiro L et al. (2013) Effect of household-based drinking water chlorination on diarrhoea among children under five in Orissa, India: a double-blind randomised placebo-controlled trial. PLoS Medicine 10, e1001497.

Boschi-Pinto C, Velebit L \& Shibuya K (2008). Estimating child mortality due to diarrhoea in developing countries. Bull World Health Organ 86, 710-717.

Brown J \& Clasen T (2012) High adherence is necessary to realize health gains from water quality interventions. PLoS One 7, e36735.

Cairncross S, Hunt C, Boisson S et al. (2010) Water, sanitation and hygiene for the prevention of diarrhoea. International Journal of Epidemiology 39(Suppl. 1), i193-i205.

Clasen T, Roberts I, Rabie T, Schmidt W \& Cairncross S (2006). Interventions to improve water quality for preventing diarrhoea. Cochrane Database Systematic Review 3, CD004794.

Clasen T, Boeston K, Boisson S et al. (2010) Interventions to improve excreta disposal for the prevention of diarrhoeal disease (Cochrane Review). Cochrane Database Systematic Review 6, CD007180.

Colford JM, Rees JR, Wade T et al. (2002) Participant blinding and gastrointestinal illness in a randomized, controlled trial of 
an in-home drinking water intervention. Emerging Infectious Diseases 8, 29-36.

Dangour AD, Watson L, Cumming O et al. (2013) Interventions to improve water quality and supply, sanitation and hygiene practices, and their effects on the nutritional status of children. Cochrane Database Systematic Review 8, CD009382.

Engell RE \& Lim SS (2013) Does clean water matter? An updated meta-analysis of water supply and sanitation interventions and diarrhoeal diseases. Lancet 381, S44. doi: 10.1016/ S0140-6736(13)61298-2.

Enger KS, Nelson KL, Rose JB \& Eisenberg JN (2013) The joint effects of efficacy and compliance: a study of household water treatment effectiveness against childhood diarrhea. Water Research 47, 1181-1190.

Ezzati M, Lopez AD, Rodgers A, Van der Hoorn S \& Murray CJ (2002) Comparative Risk Assessment Collaborating Group. Selected major risk factors and global and regional burden of disease. Lancet 360, 1347-1360.

Ezzati M, Lopez AD, Rodgers A \& Murray CJL (2004). Comparative Quantification of Health Risks: Global and Regional Burden of Diseases Attributable to Selected Major Risk Factors. WHO, Geneva.

Farr W (1885) Vital Statistics, a Memorial Volume of Selections from the Reports and Writings of William Farr London: Sanitary Institute, 1885: xi. (Reprinted Scarecrow Press, New York, 1975).

Fewtrell L, Kaufmann R, Kay D et al. (2005) Water, sanitation, and hygiene interventions to reduce diarrhoea in less developed countries: a systematic review and meta-analysis. The Lancet Infectious Diseases 5, 42-52.

Fleischer NL, Fernald LCH \& Hubbard AE (2010) Estimating the potential impacts of intervention from observational data: methods for estimating causal attributable risk in a cross-sectional analysis of depressive symptoms in Latin America. Journal of Epidemiology and Community Health 64, 16-21.

Greenland S \& Drescher K (1993) Maximum likelihood estimation of the attributable fraction from logistic models. Biometrics $49,865-872$.

Hellard ME, Sinclair MI, Forbes AB \& Fairley CK (2001) A randomized, blinded, controlled trial investigating the gastrointestinal health effects of drinking water quality. Environmental Health Perspectives 109, 773-778.

Hubbard AE \& van der Laan MJ (2008) Population intervention models in causal inference. Biometrika 95, 35-47.

Humphrey J (2009) Child undernutrition, tropical enteropathy, toilets, and handwashing. Lancet 374, 1032-1035.

Hunter PR (2009) Household water treatment in developing countries: comparing different intervention types using metaregression. Environmental Science and Technology 43, 89918997.

Huttly SR, Morris SS \& Pisani V (1997) Prevention of diarrhoea in young children in developing countries. Bulletin of the World Health Organization 75, 163-174.

Jain S, Sahanoon OK, Blanton E et al. (2010) Sodium dichloroisocyanurate tablets for routine treatment of household drinking water in periurban Ghana: a randomized controlled trial. American Journal of Tropical Medicine and Hygiene 82, 16-22.

Kirchhoff LV, McClelland KE, Do Carmo Pinho M, Araujo JG, De Sousa MA \& Guerrant RL (1985) Feasibility and efficacy of in-home care chlorination in rural North-eastern Brazil. The Journal of Hygiene 94, 173-180.

Lim SS, Vos T, Flaxman AD et al. (2012) A comparative risk assessment of burden of disease and injury attributable to 67 risk factors and risk factor clusters in 21 regions, 1990-2010: a systematic analysis for the Global Burden of Disease Study 2010. Lancet 380, 2224-2260.

Lin A, Arnold BF, Afreen S et al. (2013) Environmental enteropathy and impaired growth in rural Bangladesh. American Journal of Tropical Medicine and Hygiene 89, 130-137.

Lopez AD (2005) The evolution of global burden of disease framework for disease, injury and risk factor quantification: developing the evidence base for national, regional and global public health action. Globalization and Health 1, 5.

Lozano R, Naghavi M, Foreman K et al. (2012) Global and regional mortality from 235 causes of death for 20 age groups in 1990 and 2010: a systematic analysis for the Global Burden of Disease Study 2010. Lancet, 380, 2095-2128.

Muñoz ID \& van der Laan M (2012) Population intervention causal effects based on stochastic interventions. Biometrics 68 , 541-549.

Murray CJL (1996) Rethinking DALYs. In: The Global Burden of Disease. (eds CJL Murray AD Lopez) Harvard University Press on behalf of the World Health Organization and the World Bank, Cambridge, MA.

Murray CJL \& Lopez AD (1996) The Global Burden of Diseases. Harvard School of Public Health, WHO, World Bank, Cambridge, MA.

Murray CJ \& Lopez AD (1997) Global mortality, disability, and the contribution of risk factors: Global Burden of Disease Study. Lancet 349, 1436-1442.

Murray CJL \& Lopez AD (1999) On the comparable quantification of health risks: lessons from the Global Burden of Disease Study. Epidemiology 10, 594-605.

Murray CJL, Ezzati M, Flaxman AD et al. (2012) GBD 2010: design, definitions, and metrics. Lancet 380, 2063-2066, Appendix.

Onda K, Lobuglio J \& Bartram J (2013) Global access to safe water: accounting for water quality and the resulting impact on MDG progress. World Health \& Population 14, $32-44$.

Pruss A, Kay D, Fewtrell L \& Bartram J (2002) Estimating the burden of disease from water, sanitation and hygiene at a global level. Environmental Health Perspectives 110, 537-542.

Pruss-Ustun A, Kay D, Fewtrell L \& Bartram J (2002). Unsafe Water, Sanitation and Hygiene. Comparative Quantification of Health Risks, Chap. 16. World Health Organization, Geneva.

Quick RE, Venczel LV, Mintz ED et al. (1999) Diarrhoea prevention in Bolivia through point-of-use water treatment and 
T. Clasen et al. Estimating the impact of unsafe water

safe storage: a promising new strategy. Epidemiology and Infection 122, 83-90.

Robins JM, Hernan MA \& Brumback B (2000) Marginal structural models and causal inference in epidemiology. Epidemiology 11, 550-560.

Savović J, Jones H, Altman D et al. (2012) Influence of reported study design characteristics on intervention effect estimates from randomised controlled trials: combined analysis of metaepidemiological studies. Health Technology Assessment 16, $1-82$.

Schmidt WP \& Cairncross S (2009) Household water treatment in poor populations: is there enough evidence for scaling up now? Environmental Science and Technology 43, 986-992.

Semenza JC, Roberts L, Henderson A, Bogan J \& Rubin CH (1998) Water distribution system and diarrhoeal disease transmission: a case study in Uzbeckistan. American Journal of Tropical Medicine and Hygiene 59, 941-946.

van der Laan M \& Rose S (2011) Targeted Learning: Causal Inference for Observational and Experimental Data. Springer, New York.
Waddington H, Snilstveit B, White H \& Fewtrell L. (2009) Water, Sanitation and Hygiene Interventions to Combat Childhood Diarrhoea in Developing Countries. 3ie. World Bank, New Delhi, India. Investing in health: World Development Report 1993, Washington DC: World Bank.

Watts C \& Cairncross S (2012) Should the GBD risk factor rankings be used to guide policy? Lancet 380, 2060-2061.

WHO (2008). The Global Burden of Disease: 2004 Update. World Health Organization, Geneva.

WHO (2009) Global Health Risks: Mortality and Burden of Disease Attributable to Selected Major Risks. World Health Organization, Geneva.

WHO/UNICEF (2012) Progress on Drinking Water and Sanitation: 2012 Update. World Health Organization, Geneva and UNICEF, New York.

Wood L, Egger M, Lotte Gluud L et al. (2008) Empirical evidence of bias in treatment effect estimates in controlled trials with different interventions and outcomes: meta-epidemiological study. BMJ 336, 6Fi01-6Fi60.

World Bank (1993) World Development Report 1993: Investing in Health, Vol. 1. World Bank, Washington, DC.

Corresponding Author Thomas Clasen, 1518 Clifton Road, Mailstop 1518-002-2BB, Atlanta, GA 30322, USA. Tel.: +1 404 4273480; E-mail: tfclasen@emory.edu 